\title{
Nutrition behaviours among female students and their association with BMI, self-perceived health status, Saudi Arabia.
}

\author{
Suneetha Epuru ${ }^{1}$, Afaf Obaid Shaib AlMuqrn ${ }^{2}$ \\ ${ }^{1,2}$ (Clinical Nutrition Department, College of Applied Medical Sciences / University of Hail, Saudi Arabia)
}

\begin{abstract}
:
Background: Nutrition-related diseases are a leading cause of morbidity and mortality in the KSA. Limited information exists regarding nutrition and health behaviours of college-aged females.

Objectives: The aim of this study is to assess the current nutrition behaviours in a sample of subjects from the University of Hail.

Methods: This study involved 241 students enrolled in UOH, female campus. A confidential survey requesting self -reported information on nutrition behaviours, health status, weight and height was collected using a standardised questionnaire.

Results: Mean age and BMI was $20.68 \pm 1.75$ and $23.30 \pm 4.50$. The majority of subjects reported that their perceived health is good (76 \%) and physically they feel strong (63\%).Only around $19 \%$ eat fresh fruits, $30 \%$ drink milk, $25 \%$ drink fresh juices 5 or more days in a week while atleast $50 \%$ drink soft drinks, $49 \%$ eat chips, $67 \%$ eat chocolates, $44 \%$ eat desserts/sweets, $32 \%$ eat fast foods. Obesity is significantly high in those subjects with high chips consumption habit $\left(\chi^{2}=14.401 ; p=0.025\right)$ and less water consuming habit $\left(\chi^{2}=\right.$ 14.401; $p=0.025$ ).
\end{abstract}

Conclusion: The results of this study indicate that study subjects nutrition behaviours are health risky. Keywords: Nutrition Behaviours, Saudi Arabia, Self-rated Health, Young Adults.

\section{Introduction}

College students between the ages of 18 and 24 years are in a transition phase between adolescence and adulthood which help them gain new experiences and personal freedom as well as develop a sense of identity ${ }^{1}$. However, given their hectic schedules the newly gained freedom from parental influence often results in the tendency to engage in unhealthy dieting, meal skipping, and fast food consumption which can likely increase their future risk for non-communicable diseases and quality of life ${ }^{2}$.These findings emphasise the importance of establishing good eating habits from an early age ${ }^{3}$.

Nutrition knowledge and converting this into practice are critical to improve health and nutrition in a sustainable way. Young adulthood is the time to learn and adopt healthy habits to avoid many health and nutritional problems later in life ${ }^{4}$. They have more easy access to health and nutrition information through colleges, recreational activities, and mass media than they have later in their lives ${ }^{5}$. Particularly, health and nutrition knowledge and healthy habits of female young adults will have critical roles to play in maintaining future family health and nutrition.

There have been a variety of international dietary recommendations for reducing health risks and live a long healthy life. During the past decades, dietary recommendations have tended to shift from nutrients to an overall dietary pattern, with emphasis on varied consumption and with specific attention to certain food groups (eg. limiting consumption of red and processed meats, increase in intake of fruits, vegetables, low fat dairy products and whole-grain foods). For example, 2010 US Dietary Guidelines ${ }^{6}$ and food-group recommendations have been extended and advise a shift in food intake to a more plant-based diet that emphasizes fruit, vegetables, legumes, whole grains, nuts, and seeds. In addition, increased intakes of seafood and low-fat dairy products and consumption of only moderate amounts of lean meats, poultry, and eggs were likewise been recommended ${ }^{6}$. Recommendations are ultimately intended to guide the public to a healthful diet. For the public compared with nutrient recommendations, food recommendations are easier to implement since they are more stable over a period of time. However, research needs to focus on how much of these recommendations correspond to actual changes in dietary intakes in the general population and whether changes are different in young adult groups.

In view of the foregoing discussions, this study titled "nutrition behaviour patterns among the college students of University of Hail" was undertaken with the following objectives.

\subsection{Objectives}

- To assess the current nutrition and dietary practices in a sample of subjects from the University of Hail.

- To study the relationship between food group recommendations and nutrition behaviour patterns.

- To study the association of previous nutrition knowledge on nutrition behaviours. 


\section{Methodology}

A cross sectional survey was planned and conducted to assess the nutrition behaviour patterns among a random female sample of 241 subjects of University of Hail.

\subsection{Design, Sample and Data Collection}

The study design was a cross-sectional survey and was conducted at the University of Hail (UOH) during the winter $2012-13$ semester. Approximately, a random sample size of 241 sample was enrolled in the study from different colleges in female campus. University of Hail research committee approved the study. No personal identification details were collected protecting privacy and allowing for anonymous and voluntary participation. The inclusion criteria included current registration with University of Hail as students, absence of chronic illness, and knowledge of their height, weight information and acceptance of informed consent form. The exclusion criteria included those with less than 18 years and those who are following any special diets, pregnant and lactating mothers.

\subsection{Anthropometry}

The subjects were asked to record their self-reported height and weight information on the questionnaire. Those who were not aware of the information were excluded from the participation of the study. Body Mass Index (BMI) was calculated as the ratio of weight (kilograms) to the square of height (meters). Weight status was classified into four categories: underweight (BMI $\leq 18.5$ ), normal weight (BMI between 18.5 -24.9), overweight (BMI between 25-29.9), and obese (BMI $\geq 30)^{7}$.

\subsection{Assessment Tool}

The questionnaire requested information on socio-demographic and anthropometric information which included age, gender, college, height and weight followed by questions related to dietary behaviours, preferences and knowledge. The participants were asked regarding their current dietary practices which were tailor made to be more suitable with the dietary and food culture patterns of the Saudi students.

Validation of the Questionnaire: For content validity (back to back translation), the questionnaire was initially translated into Arabic and then converted back to English and pre-tested for question accuracy and clarity.

\subsection{Statistical Analysis}

The data set was cleaned and edited for inconsistencies. Missing data were not statistically computed. Statistical analyses were performed using the Statistical Package for Social Sciences (version 16.0, SPSS, Inc) software. Descriptive statistics such as means and standard deviations were calculated for the continuous variables and frequencies for qualitative data. Associations were established using chi-square analysis. All reported $P$ values were made on the basis of 2 -sided tests and compared to a significance level of 5\%; differences were considered statistically significant at $\mathrm{P}<0.05$ or $\mathrm{P}<0.01$.

\section{Results}

Table 1 presents the demographic and anthropometric profile of the study subjects $(\mathrm{n}=241)$. The total mean age \pm SD was $20.68 \pm 1.75$ years (range 18-24) and mean \pm SD BMI was $23.30 \pm 4.50$ which was closer towards the upper cut-off of the international normal range (BMI 18.5 to 25). The Table also presents BMI group distribution among the study population. Nearly $23 \%$ and $7 \%$ of study population were overweight and obese respectively while around $12 \%$ were underweight indicating the poor nutrition status among the study population.

Table 2 presents the meal patterns of study subjects. Only $45.6 \%$ suggested that they eat breakfast daily and nearly $42 \%$ eat breakfast less than 4 days in a week. Among those who eat breakfast, majority eat at college canteen daily rather than at home. Only around $35 \%$ suggested they take regularly all three meals (breakfast, lunch and dinner) in a day and $39 \%$ eat three meals less than 4 days in a week which indicates the prevalence of health risk behaviours in these subjects. Around $58 \%$ eat from restaurants atleast less than 4 days in a week while college canteen is an important part in providing one part of the meal for atleast $40.9 \%$ of the subjects. Around $50.4 \%$ percent eat atleast one meal with friends while a majority of $63.9 \%$ eat with family a minimum of one meal daily.

Table 3 suggests dietary behaviours of the study subjects. Only around $28 \%$ eat fresh vegetables 5 or more days in a week. Similarly only around $19 \%$ eat fresh fruits, $30 \%$ drink milk, $25 \%$ drink fresh juices 5 or more days in a week. In contrast, study subjects prefer foods like soft drinks, chips, chocolates, sweets etc. Atleast $50 \%$ drink soft drinks, $49 \%$ eat chips, $67 \%$ eat chocolates, $44 \%$ eat desserts/sweets and $32 \%$ eat fast foods on regular basis 5 or more days in a week. Fish is eaten atleast by $39 \%$ once or twice in a week. Canned foods and processed foods are also highly consumed. The study results indicate atleast $29 \%$ eat canned foods, 
$33.5 \%$ eat processed foods and $33 \%$ eat readymade yogurt products 5 or more days in a week. Only around 19 $\%$ of the subjects indicted that they drink daily minimum 8 glasses of water.

Table 4 presents perceptions and practices in study population. Around $12 \%$ of the study population had indicated that they are suffering from constipation from majority days while nearly $23 \%$ suggest that they sleep less than 8 hours for atleast 4 or less days in a week. Nearly $76 \%$ perceive their health to be "good" while another $63 \%$ describe their physical strength as "strong". Around $27 \%$ feel fresh throughout the day, while 16 $\%$ feel attentive. A whopping $21 \%$ describe themselves as tired always and another $25 \%$ feel sleepy during the day.

Table 5 present the associations of BMI with chips consumption, minimum 8 glasses of water consumption and body state perceptions and indicates that obesity is significantly high in those subjects with high chips consumption habit $\left(\chi^{2}=4.296 ; \mathrm{p}=0.038 ; \mathrm{OR}=1.814\right.$ (95\% CI- 1.030- 3.197) and less water consuming habit $\left(\chi^{2}=6.688 ; p=0.010 ; O R=1.261(95 \%\right.$ CI- $1.033-1.541)$.

Table 5 also presents the associations of perception of strong physical strength with fresh fruit consumption and soft drink consumption and suggest that perception of strong physical strength is significantly high in those subjects with high fresh fruit consumption habit $\left(\chi^{2}=4.616, p=0.032 ;\right.$ OR $=2.280$ (95\% CI- 1.0604.902) and less soft drink consuming habit $\left(\chi^{2}=7.400, \mathrm{p}=0.007\right.$; OR=1.613 (95\% CI- 1.132- 2.299).

The associations of perception of good health with minimum $8 \mathrm{hrs}$. of sleep habit and a minimum of 3 meals a day were also presented in Table 5 and indicates that perception of good health is significantly high in those subjects with good sleep habits $\left(\chi^{2}=5.136, p=0.023\right.$; OR=2.418 (95\% CI- 1.108- 5.276) and meal habits $\left(\chi^{2}=6.245, \mathrm{p}=0.012 ; \mathrm{OR}=2.751(95 \%\right.$ CI- 1.216- 6.223).

The results also suggested associations of body state perception with minimum 8 hrs. of sleep habit, breakfast habit and eating fresh vegetables most days (Table 6). The body state perception is significantly better in those subjects with good sleep habits $\left(\chi^{2}=7.085, \mathrm{p}=0.013\right)$ breakfast habit $\left(\chi^{2}=10.728, \mathrm{p}=0.03\right)$ and high fresh fruit consumption habit $\left(\chi^{2}=7.921, \mathrm{p}=0.02\right)$.

\section{Discussion}

The objective of this cross-sectional study was to identify nutrition behaviours with an emphasis on the relationship between perceptions of their health and body state. As expected, female gender were associated with poor dietary behaviours like eating high amounts of chips, chocolates, sweets, soft drinks and fast foods while were significantly at risk for skipping breakfast, meals and less consumption of protective food like milk, fruits and vegetables.

Research supports increasing evidence for the need to emphasize strengthening good health and dietary behaviours among young adults for optimum health and well-being. They represent a transition period from adolescence to adulthood and are vulnerable to poor lifestyle choices and therefore require emphasis regarding their health risk behaviours in view of improving their future health. In general, young adults are frequently identified as being at risk for health risk lifestyle behaviours given their hectic schedules in college and private life.Young adults are potentially at risk for poor dietary choices largely because of their very high nutrient demands coupled with incompatibility of their range of preferred foods and patterns of eating. Their physiological demands drive appetite while meals are dependent mostly on convenience foods and influence of peer groups ${ }^{8}$.

The results of the present study are comparable with other studies reported form Saudi Arabia and neighbouring countries with a similar cultural background. The available limited research from Saudi Arabia focusing on dietary habits in young adults raise alarm for increasing fast food culture and poor life style choices 9-12. A recent national level nutrition survey in Saudi Arabia has indicated that young adults are undergoing nutrition transition from traditional foods to preference over eating non-nutritional and high calorie snacks like fried foods and carbonated drinks commonly in day to day life ${ }^{13}$. These changes reflect the shifting socioenvironmental conditions which can predispose young adults to obesity.

In the present study, for subjects reporting perception of good health, strong physical feeling and fresh and attentive mind states were significantly associated with health promoting habits like regular meal and breakfast habits, fresh fruit and vegetable consumption and good sleep habits implying the need for awareness of the role of dietary behaviours on health among young people. These relations are in support of previous similar findings ${ }^{14-17}$. Sleep quality, although differently assessed in various studies, seems to have an important relationship with feeling of fresh and attentive. These results are in agreement with previous studies ${ }^{18-20}$.

BMI is another important indicator of health status. Research suggests transition period of adolescence to adulthood between the ages of 18 to 29 can cause greatest increases in obesity ${ }^{21}$ and particularly young women are at higher risk ${ }^{9,10}$. The present study projects high prevalence of obesity and also association with dietary behaviours like high chips consumption and drinking less water emphasising the need for giving due recognition to the nutrient. Also associations between BMI and perceptions of mental state indicate the link of obesity with tiredness and fatigue which are in agreement with previous studies ${ }^{22}$. The present study trends 
indicate that the perceptions about their health status suggest low confidence level especially among the obese subjects highlighting the importance of urgent need for proper nutrition education intervention in this population.

The present study has a number of limitations. Firstly, the design of the study is cross-sectional which may probably not infer causal associations among measured variables. Repeated studies with larger sample size are required in future to test the reliability of the present results. Secondly, anthropometric measurements, dietary habit information and health, physical and mental status reporting was based on self-report which can increase the likelihood of bias. Thirdly, for water consumption measurement, seasonal influences (data was collected during winter period) could be the reason for high prevalence of subjects with less than minimum amount of drinking water habit. Finally, we did not include other life style factors like physical activity which can influence health status perception like physical activity.

\section{Conclusions}

Results from this study highlight the importance of early identification of the health risk behaviors in young adults and the need to promote healthy dietary interventions. A higher percent prevalence of health risk behaviours like breakfast skipping, low intake of fruits, vegetables, milk coupled with high soft drink, chips, chocolate and fast food culture among the study subjects indicates an alarming situation towards their future health status. Health education campaigns, which raise the knowledge on risk factors and unhealthy behaviors among youth's can educate and self-guard young adults.

\section{References}

[1]. Franko DL, Cousineau TM, Trant M, Green TC, Rancourt D, Thompson D, et al. Motivation, self-efficacy, physical activity and nutrition in college students: Randomized controlled trial of an internet-based education program. Prev Med. 2008;47(4):369-377.

[2]. Silliman K, Rodas-Fortier K, Neyman M: A survey of dietary and exercise habits and perceived barriers to following a healthy lifestyle in a college population. Californian J Health Promot. 2004;2(2):10-19.

[3]. White S, Park YS, Isreal T, Cordero ED. Longitudinal evaluation of peer health education on a college campus: Impact on health behaviors. J Am Coll Health. 2009;57(5):497-505.

[4]. Nelson MC, Story M, Larson NI, Neumark-Sztainer D, Lytle LA: Emerging adulthood and college-aged youth: An overlooked age for weight-related behavior change. Obes 2008;16(10):2205-2211.

[5]. Ferrara CM. The college experience: physical activity, nutrition, and implications for intervention and future research. JPEonline. 2009; 12(1): 23-35

[6]. Report of the Dietary Guidelines Advisory Committee on the Dietary Guidelines for Americans, 2010, to the Secretary of Agriculture and the Secretary of Health and Human Services. Washington, DC: US Department of Agriculture, Agricultural Research Service, 2010. Available from: http://www.cnpp.usda.gov/DGAs2010-DGACReport.htm (accessed on 31 January 2013).

[7]. Clinical guidelines on the identification, evaluation, and treatment of overweight and obesity in adults-the evidence report. National Institutes of Health Obesity Research 1998;(Suppl 2):51S-209S

[8]. Simon Langley-Evans. Nutrition: A Lifespan Approach: Nutrition and Adolescence. Edition 3, WileyPublishers, 2009.

[9]. Rasheed P, Abou-Hozaifa BM, Kahn A. Obesity among young Saudi female adults: a prevalence study on medical and nursing students. Public Health. 1994; 108(8): 289-294.

[10]. Al-Rethaiaa AS, Fahmy AE, Al-Shwaiyat NM. Obesity and eating habits among college students in Saudi Arabia: a cross sectional study. Nutr J. 2010; 19 (9): 9-39.

[11]. Al-Hazzaa H. The public health burden of physical inactivity in Saudi Arabia.Journal of Family and Community Medicine. 2004; 11: 45-51.

[12]. Al-Nuaim AA, Al-Nakeeb Y, Lyons M, Al-Hazzaa HA, Nevill A, Collins P, and Duncan MJ. The Prevalence of Physical Activity and Sedentary Behaviours Relative to Obesity among Adolescents from Al-Ahsa, Saudi Arabia: Rural versus Urban Variations.J. of Nut and Met. 2012; 2012: 417589.

[13]. Al-Nozha MM, Al-Mazrou YY, Al-Maatouq MA, Arafah MR, Khalil MZ, Khan NB, Al-Marzouki K, Abdullah MA, Al-Khadra AH, Al-Harthi SS, Al-Shahid MS, Al-Mobeireek A, Nouh MS. Obesity in Saudi Arabia. Saudi Med J. 2005; 26: 824-29.

[14]. Halford C, Ekselius L, Anderzen I, Arnetz B, Svärdsudd K: Self-rated health, lifestyle, and psychoendocrine measures of stress in healthy adult women. Ups J Med Sci. 2010;115(4):266-74.

[15]. Girón P: Determinants of self-rated health in Spain: differences by age groups for adults. Eur J Public Health. 2010; Feb;22(1):3640. doi: 10.1093/eurpub/ckq133. Epub 2010 Oct 5.

[16]. Tsai J, Ford ES, Li C, Zhao G, Pearson WS, Balluz LS: Multiple healthy behaviors and optimal self-rated health: findings from the 2007 Behavioral Risk Factor Surveillance System Survey. Prev Med. 2010;51(3-4):268-74.

[17]. Daniilidou NV, Gregory S, Kyriopoulos JH, Zavras DJ. Factors associated with self-rated health in Greece: a population-based postal survey. Eur J Public Health. 2004;14(2):209-11.

[18]. Prosper MH, Moczulski VL, Qureshi A: Obesity as a predictor of self-rated health. Am J Health Behav. 2009;33(3):319-29.

[19]. Molarius A, Berglund K, Eriksson C, Lambe M, Nordström E, Eriksson HG, Feldman I. Socioeconomic conditions, lifestyle factors, and self-rated health among men and women in Sweden. Eur J Public Health. 2007;17(2):125-33.

[20]. Darviri C, Artemiadis AK, Tigani X, Alexopoulos EC. Lifestyle and self-rated health: a cross-sectional study of 3,601 citizens of Athens, Greece. BMC Public Health. 2011; Aug 4;11:619. doi: 10.1186/1471-2458-11-619

[21]. Ng SW, Zaghloul S, Ali Hi, Harrison G and Popkin BM. The prevalence and trends of overweight, obesity and nutrition-related non-communicable diseases in the Arabian Gulf States. Obesity Reviews. 2011; 12:1-13.

[22]. Harrington J, Perry IJ, Lutomski J, Fitzgerald AP, Shiely F, McGee H, Barry MM, Van Lente E, Morgan K, Shelley E: Living longer and feeling better: healthy lifestyle, self-rated health, obesity and depression in Ireland. Eur J Public Health. 2010;20(1):915 . 
Table 1: Demographic and Anthropometric Profile of the Study Population

\begin{tabular}{|lcccc|}
\hline Variables & Minimum & Maximum & Mean & $\begin{array}{c}\text { Standard } \\
\text { Deviation }\end{array}$ \\
Age (years) & & & & 20.68 \\
Weight $(\mathbf{k g})$ & 18 & 24 & 58.98 & 1.75 \\
Height $(\mathbf{c m})$ & 30 & 107 & 159.10 & 6.59 \\
BMI $(\mathbf{k g} / \mathbf{m} 2)$ & 143 & 187 & 23.30 & 4.50 \\
BMI & 14.47 & 43.41 & Over weight & Obese \\
Percentage & Underweight & Normal & 22.82 & 7.05 \\
\hline
\end{tabular}

Table 2: Meal Patterns of Study Subjects (\%)

\begin{tabular}{|c|c|c|c|c|c|}
\hline Question & Daily & $\begin{array}{l}\text { 1-2 days in a } \\
\text { week }\end{array}$ & $\begin{array}{l}\text { 3-4 days in } \\
\text { a week }\end{array}$ & $\begin{array}{l}\text { 5-6 days in a } \\
\text { week }\end{array}$ & None \\
\hline Eat breakfast & 45.60 & 24.70 & 17.20 & 8.40 & 4.20 \\
\hline Eat breakfast at home & 20.70 & 36.10 & 15.80 & 5.40 & 22.00 \\
\hline Eat breakfast at college canteen & 39.00 & 20.80 & 22.50 & 9.50 & 8.20 \\
\hline Eat three major meals & 35.40 & 24.00 & 14.80 & 12.20 & 13.50 \\
\hline Eat in restaurant & 11.00 & 37.90 & 20.70 & 15.40 & 15.00 \\
\hline Eat in college canteen & 40.90 & 17.80 & 15.20 & 11.70 & 14.30 \\
\hline Eat with friends at least one meal & 50.40 & 22.20 & 13.70 & 10.30 & 3.40 \\
\hline Eat with family at least one meal & 63.90 & 15.90 & 12.30 & 4.00 & 4.00 \\
\hline
\end{tabular}

Table 3: Dietary Behaviours of Study Subjects (\%)

\begin{tabular}{|lccccc|}
\hline \multicolumn{1}{c}{ Question } & Daily & $\begin{array}{c}\text { 1-2 days in a } \\
\text { week }\end{array}$ & $\begin{array}{c}\text { 3-4 days in } \\
\text { a week }\end{array}$ & $\begin{array}{c}\text { 5-6 days in a } \\
\text { week }\end{array}$ \\
Eat fresh vegetables & & 31.20 & 20.30 & 13.00 & None \\
Eat fresh fruits & 14.70 & 43.10 & 24.10 & 10.30 & 20.80 \\
Drink milk & 8.60 & 27.50 & 18.50 & 7.70 & 13.80 \\
Drink fruit juices & 22.30 & 38.60 & 22.00 & 8.90 & 14.00 \\
Drink soft drinks & 16.10 & 22.80 & 16.80 & 14.70 & 9.90 \\
Eat chips & 35.80 & 29.10 & 20.70 & 9.70 & 2.50 \\
Eat chocolates & 38.00 & 18.90 & 12.20 & 15.10 & 2.50 \\
Eat desserts/ sweets & 51.30 & 30.30 & 20.50 & 13.20 & 5.10 \\
Eat fast foods & 30.80 & 37.90 & 23.00 & 15.70 & 6.80 \\
Eat fish & 16.60 & 38.80 & 9.10 & 10.30 & 3.80 \\
Eat canned foods & 2.60 & 30.70 & 24.60 & 11.20 & 15.40 \\
Eat processed foods & 20.60 & 30.80 & 22.30 & 6.90 & 13.40 \\
Eat yogurt based foods & 22.30 & 34.50 & 20.70 & 1.60 & 11.60 \\
Drink minimum eight glasses of & 26.30 & 27.00 & 14.80 & & 32.10 \\
water & 18.60 & & & & \\
\hline
\end{tabular}

Table 4: Perceptions and practices of Study Subjects (\%)

\begin{tabular}{|c|c|c|c|c|c|}
\hline Question & Daily & $\begin{array}{l}\text { 1-2 days in } \\
\text { a week }\end{array}$ & $\begin{array}{c}\text { 3-4 days } \\
\text { in a week }\end{array}$ & $\begin{array}{l}\text { 5-6 days in a } \\
\text { week }\end{array}$ & None \\
\hline Suffer from constipation & 5.20 & 18.10 & 9.50 & 6.90 & 60.30 \\
\hline \multirow[t]{2}{*}{ Sleep minimum for eight hours daily } & 43.70 & 19.70 & 12.20 & 9.20 & 15.10 \\
\hline & Good & Very Good & Poor & Very Poor & I can't say \\
\hline \multirow[t]{2}{*}{ Describe my usual health as } & 52.10 & 34.00 & 8.40 & 1.30 & 4.20 \\
\hline & Strong & $\begin{array}{c}\text { Very } \\
\text { Strong }\end{array}$ & Weak & Very Weak & I can't say \\
\hline \multirow[t]{2}{*}{ Describe my usual physical strength as } & 54.20 & 8.40 & 23.10 & 3.80 & 10.50 \\
\hline & Tired & Fresh & Attentive & Sleepy & I can't say \\
\hline Describe my usual mental state as & 20.70 & 27.00 & 15.80 & 24.90 & 11.60 \\
\hline
\end{tabular}

Table 5: Health and Nutrition Practices vs. Obesity and Health Perceptions of Study Subjects (\%)

\begin{tabular}{|c|c|c|c|c|}
\hline Obesity & Obese & Non Obese & Chi-square & $\begin{array}{l}\text { Odd's Ratio } \\
(95 \% \text { CI })\end{array}$ \\
\hline Eat chips $\leq 4$ days in a week & 37.50 & 47.88 & 4.296 & 1.814 \\
\hline Eat chips 5 or days in a week & 62.50 & 52.12 & $(\mathrm{p}=0.038)$ & $(1.030-3.197)$ \\
\hline $\begin{array}{l}\text { Drink Minimum } 8 \text { glasses of water } \leq 4 \text { days } \\
\text { in a week }\end{array}$ & 37.68 & 21.43 & $\begin{array}{c}6.688 \\
(p=0.010)\end{array}$ & $\begin{array}{c}1.261 \\
(1.033-1.541)\end{array}$ \\
\hline $\begin{array}{l}\text { Drink Minimum } 8 \text { glasses of water } 5 \text { or days } \\
\text { in a week }\end{array}$ & 62.32 & 78.57 & & \\
\hline Physical State Perceptions & Feeling Weak & Feeling Strong & & \\
\hline
\end{tabular}


Nutrition behaviours among female students and their association with BMI, self-perceived health

\begin{tabular}{|c|c|c|c|c|}
\hline Eat fresh fruits $<4$ days in a week & 40.86 & 59.14 & 4.616 & 2.280 \\
\hline Eat fresh fruits 5 or days in a week & 23.26 & 76.74 & $(\mathrm{p}=0.032)$ & $(1.060-4.902)$ \\
\hline Habit of soft drinks $<4$ days in a week & 28.32 & 71.68 & 7.400 & 1.613 \\
\hline Habit of soft drinks 5 or days in a week & 45.69 & 54.31 & $(\mathrm{p}=0.007)$ & $(1.132-2.299)$ \\
\hline Health Status Perceptions & Good Health & Poor Health & & \\
\hline Minimum 8 hrs. sleep $<4$ days in a week & 81.08 & 18.92 & 5.136 & 2.418 \\
\hline Minimum 8 hrs. sleep 5 or days in a week & 91.2 & 8.8 & $(\mathrm{p}=0.023)$ & $(1.108-5.276)$ \\
\hline Eat 3 main meals $<4$ days in a week & 20.17 & 79.83 & 6.245 & 2.751 \\
\hline Eat 3 main meals 5 or days in a week & 8.4 & 91.6 & $(\mathrm{p}=0.12)$ & $(1.216-6.223)$ \\
\hline
\end{tabular}

Table 6: Mental State Perceptions and Healthy Practices of Study Subjects (\%)

\begin{tabular}{|c|c|c|c|c|c|}
\hline & Sleepy & Tired & Attentive & Fresh & Chi-square \\
\hline Minimum 8 hrs. sleep $<4$ days in a week & 28.17 & 23.94 & 14.08 & 19.72 & 7.085 \\
\hline Minimum 8 hrs. sleep 5 or days in a week & 23.95 & 19.76 & 16.77 & 30.54 & $(\mathrm{p}=0.013)$ \\
\hline Eat breakfast $<4$ days in a week & 32.11 & 22.94 & 14.68 & 18.35 & 10.728 \\
\hline Eat breakfast 5 or days in a week & 18.90 & 19.69 & 17.32 & 34.65 & $(\mathrm{p}=0.03)$ \\
\hline Eat vegetables $<4$ days in a week & 28.05 & 22.56 & 14.63 & 23.78 & 7.921 \\
\hline Eat vegetables 5 or days in a week & 15.63 & 20.31 & 18.75 & 37.50 & $(\mathrm{p}=0.02)$ \\
\hline
\end{tabular}

